\title{
FINITE ELEMENT ERROR ESTIMATES FOR SUBSONIC FLOW
}

\author{
S. S. CHOW' ${ }^{1}$ AND G. F. CAREY ${ }^{1}$
}

(Received 30 September 1985; revised 21 July 1986 and 23 October 1986)

\begin{abstract}
Error estimates are derived for a finite element analysis of plane steady subsonic flows described by the full potential equation. The analysis is based on the use of the theory of variational inequalities to accomodate the subsonic flow constraint and leads to a suboptimal estimate relative to that obtained for linear potential flow. We then consider an alternative dual formulation of the problem and obtain an optimal estimate subject to reasonable regularity assumptions.
\end{abstract}

\section{Introduction}

Plane steady compressible flow problems are of practical interest in analysis of nozzle flows, cascade flows in turbomachines and, particularly, exterior flow past airfoils. Let us consider a profile such as an airfoil with uniform incident flow in the far field. If the incident velocity is sufficiently moderate, the flow is everywhere subsonic (subcritical) and the governing equation is elliptic. Above a critical incident velocity, the flow becomes transonic with a local imbedded supersonic "pocket" adjacent to the airfoil and is of mixed type-elliptic in the subsonic flow and hyperbolic in the local supersonic flow region. At sufficiently high incident velocities, the supersonic region may be terminated by an imbedded shock discontinuity. For the subsonic flow case, and even for slightly supercritical

\footnotetext{
${ }^{1}$ Department of Aerospace Engineering and Engineering Mechanics, The University of Texas at Austin, Austin, Texas, U.S.A.

(c) Copyright Australian Mathematical Society 1987, Serial-fee code 0334-2700/87
} 
flows including weak shocks, the flow may be assumed to remain irrotational and governed by the full potential equation $[17,19]$.

The mathematical theory for subsonic flows is relatively well developed with existence and uniqueness results established for sufficiently regular data using both classical analysis based on quasiconformal mapping and also functional analytic techniques $[1,10,11,16]$. Analytic solutions for the mixed elliptic-hyperbolic problem have been constructed via hodograph transformations, but the analysis of these flows in general is more difficult $[2,13]$ and the standard-function analytic techniques associated with elliptic problems clearly are not applicable.

Several finite element methods have been developed to compute approximate solutions to the full potential equation $[3,4,5,14,18]$. A viable finite element theory should include error estimates for the approximations and conditions on the regularity of the solution or data for convergence. While these estimates are easy to obtain for the standard linear potential flow problem, the nonlinear problem is more difficult. Very restrictive estimates have been obtained for the special case of a fictitious (non-physical) gas using techniques based on those for analysis of the minimal surface equation and applied for shock-free redesign $[6,7]$. A viable error analysis for the subsonic flow of a real gas has not previously been developed. A major complication for the error analysis of subsonic flow of a real gas stems from the mathematical restriction that, for ellipticity, the flow should be everywhere less than the speed of sound in the gas. The constraint is violated for supercritical flow, but the established error analysis techniques are then no longer applicable. This factor was overlooked in an earlier attempt [15] to provide error estimates for this problem.

In the present study, we derive error estimates for the full potential equation using the theory of variational inequalities to include the mathematical restriction that admissible velocities be subsonic. In Section 1, we define the subsonic compressible flow problem, constraint subset and variational inequality formulation for the potential solution. This is followed in Section 2.1 by the finite element approximation to the variational inequality and error analysis. Since the approximation subset is not contained in the constraint subset, the analysis leads to a suboptimal estimate. This difficulty has led us to explore an alternative dual formulation involving velocity components in Section 2.2 . The central idea is that the subsonic constraint is easier to accommodate directly in terms of the velocity components, rather than as a condition on the gradient of the potential. We can thereby avoid the difficulty in the previous formulation which led to a suboptimal estimate. However, the far-field boundary condition now enters in a more complex manner. We consider two formulations in which the far-field condition is embedded in the space and using a Lagrange multiplier, respectively. Using the latter formulation, an optimal error estimate is obtained for the dual formulation. 


\section{Analysis}

\subsection{Formulation}

Consider the steady flow of a compressible gas having density $\rho$ and velocity $q$. Conservation of mass implies

$$
\nabla \cdot(\rho \mathbf{q})=0 \text { in } \Omega
$$

where $\rho=\rho\left(q^{2}\right)$ with $q=|\mathbf{q}|$.

The adiabatic equation of state for pressure $p=k \rho^{\gamma}$ and the equation for the speed of sound, $d p / d \rho=a^{2}$, yield

$$
\rho\left(q^{2}\right)=\rho_{0}\left(1-\frac{\gamma-1}{2 a_{0}^{2}} q^{2}\right)^{1 /(\gamma-1)},
$$

where $\rho_{0}$ is the stagnation density, $a_{0}$ is the stagnation speed of sound, and $\gamma$ is the ratio of specific heats for the gas. Typically, $\gamma$ lies in the interval $(1,2)$ for a gas.

For irrotational flow $q=\nabla u$ and, using this with (1) and (2), we have the full potential equation

$$
\nabla \cdot\left(\rho\left(|\nabla u|^{2}\right) \nabla u\right)=0 \text { in } \Omega .
$$

The classical form of the subsonic flow problem is then to solve (3) subject to prescribed boundary data on the boundary $\partial \Omega=\Gamma_{0} \cup \Gamma_{1}$ of the flow domain $\Omega$ ( $\Gamma_{0}$ and $\Gamma_{1}$ are disjoint). In particular, one frequently deals with data of the form

$$
\begin{gathered}
u=g_{0} \text { on } \Gamma_{0}, \\
\rho \frac{\partial u}{\partial n}=g_{1} \quad \text { on } \Gamma_{1},
\end{gathered}
$$

representing a prescribed asymptotic far-field boundary condition on a remote boundary $\Gamma_{0}$ in the uniform flow region and a mass flux boundary condition on a profile such as an airfoil. We remark that in practice $g_{1}=0$ and we shall restrict our analysis to this case. In addition, for lifting airfoils, the circulation must be specified by means of the Kutta condition (using, for instance, a branch cut with circulation determined as a potential jump). We shall restrict our attention to flows without circulation. The flow domain $\Omega$ is a simply-connected bounded domain in $\mathbb{R}^{N}$ or a multiply-connected domain for which the flow problem can be posed on a simply-connected subdomain by appealing to physical and geometric symmetry, as in uniform flow past a symmetric airfoil at zero angle of incidence to the flow. The above problem statement is complete for subsonic flows, i.e., $q<a_{*}$, where $a_{*}$ is the critical velocity and is equal to $\sqrt{2 /(\gamma+1)} a_{0}$. For transonic flows, (3) is no longer elliptic, and one must include a further entropy condition to prevent non-physical rarefaction discontinuities. 
Rather than working with the classical statement of the subsonic full potential problem, one can instead consider a functional minimization problem on a closed convex subset $K_{\delta}$ specifying the subsonic flow constraint. We first introduce the following function spaces and notations. Let

$$
H_{\Gamma_{1}}=\left\{v \in H^{1}(\Omega) ; v=0 \text { on } \Gamma_{i}\right\}, \quad i=0,1
$$

be equipped with the $H_{0}^{1}$ norm $\|\cdot\|$. Then provided that meas $\left(\Gamma_{t}\right) \neq 0, H_{\Gamma_{t}}$ is a Hilbert space for $i=0,1$. Let $H_{\Gamma_{1}}^{\prime}$ denote the dual space of $H_{\Gamma_{1}}$ and let $\langle\cdot, \cdot\rangle$ denote the duality pairing on $H_{\Gamma_{0}}^{\prime} \times H_{\Gamma_{0}}$. The norm on $W^{m, r}(\Omega)$ is denoted by $\|\cdot\|_{m, r}$ and the norm on $L^{r}(\Omega)$ by $\|\cdot\|_{L} r$. To consider the variational formulation of (3)-(5), we first note that (3) is the Euler-Lagrange equation of the variational functional

$$
J(u)=\int_{\Omega} \int_{0}^{|\nabla u|^{2}} \frac{1}{2} \rho(t) d t d x
$$

We see from (2) that the functional $J$ is well defined for functions $u$ in $H^{1}(\Omega)$ that satisfy the constraint $|\nabla u|<a_{*}$ a.e. in $\Omega$. Thus, we consider the variational minimization problem:

Find $u \in K_{\delta}$ such that

$$
J(u)=\min J(v) ; \quad v \in K_{\delta},
$$

where

$$
K_{\delta}=\left\{v \in H^{1}(\Omega) ; v=g_{0} \text { on } \Gamma_{0} \text { and }|\nabla v| \leqslant \delta<a_{*} \text { a.e. in } \Omega\right\} .
$$

The introduction of the constant $\delta$ enables us to obtain positive, finite bounds for the density and its derivatives and to write the subsonic constraint as a non-strict inequality to facilitate the analysis. We assume $\delta$ is close to $a_{*}$. It is clear that $K_{\delta}$ is a bounded, closed and convex subset of $g_{0}+H_{\Gamma_{0}}$. In fact, $K_{\delta}$ is compact in $H^{1}(\Omega)$. We shall show later that $J$ is continuous and strictly convex on $K_{\delta}$. These results enable us to deduce the unique solvability of (7).

Taking the first variation of $J$ in (7), the variational statement for subsonic flow can be expressed as the equivalent variational inequality problem:

Find $u \in K_{\delta}$ such that

$$
\left\langle J^{\prime} u, v-u\right\rangle \geqslant 0 \quad \text { for all } v \text { in } K_{\delta},
$$

where $J^{\prime}$ is the Gâteaux derivative of $J$. Thus, $J^{\prime}$ corresponds to the full potential operator in the classical statement of problem (3) and is a non-linear operator defined according to

$$
\left\langle J^{\prime} u, v\right\rangle=\int_{\Omega} \rho \nabla u \cdot \nabla v d x
$$

for all $u$ in $K_{\delta}$ and $v$ in $H_{\Gamma_{0}}$. 
The properties of $J^{\prime}$ depend on the form of the nonlinearity-that is, on the properties of the density $\rho=\rho\left(q^{2}\right)$ in (2). We have:

(i) for $0 \leqslant q \leqslant \delta<a_{*}, \rho$ is positive bounded below and above. In fact,

$$
\rho_{0} \geqslant \rho\left(q^{2}\right) \geqslant \rho\left(\delta^{2}\right)>\rho_{0}\left(1-\frac{\gamma-1}{\gamma+1}\right)^{1 /(\gamma-1)}=\rho_{0}\left(\frac{2}{\gamma+1}\right)^{1 / \gamma} \equiv \rho_{\min }>0,
$$

since $\gamma>1$ for a real gas. Moreover, as $\rho^{\prime}=-\rho^{\gamma-1} \rho^{2-\gamma} / 2 a_{0}^{2},\left|\rho^{\prime}\right|$ is bounded above by $\rho_{0} / 2 a_{0}^{2}$.

(ii) Since

$$
\begin{aligned}
\left(\rho\left(q^{2}\right) q\right)^{\prime} & =\rho\left(q^{2}\right)+2 \rho^{\prime}\left(q^{2}\right) q^{2} \\
& =\rho^{2-\gamma}\left(q^{2}\right)\left(1-q^{2} / a^{2}\right) \rho_{0}^{\gamma-1},
\end{aligned}
$$

then

$$
\begin{array}{ll}
\left(\rho\left(q^{2}\right) q\right)^{\prime}>0 & \text { if } q \in\left[0, a_{*}\right) \\
\left(\rho\left(q^{2}\right) q\right)^{\prime}=0 & \text { if } q=a_{*} \\
\left(\rho\left(q^{2}\right) q\right)^{\prime}<0 & \text { if } q \in\left(a_{*},\left(\frac{\gamma+1}{\gamma-1}\right)^{1 / 2} a_{*}\right) .
\end{array}
$$

Furthermore, if $0 \leqslant q \leqslant \delta<a_{*}$, for $\delta$ arbitrarily close to $a_{*}$

$$
\left(\rho\left(q^{2}\right) q\right)^{\prime} \geqslant \rho^{2-\gamma}\left(\delta^{2}\right)\left(1-\delta^{2} / a_{*}^{2}\right) \rho_{0}^{\gamma-1} \geqslant C(\delta),
$$

where

$$
C(\delta)=\left(\frac{2}{\gamma+1}\right)^{(2-\gamma) /(\gamma+1)} \rho_{0}\left(1-\frac{\delta^{2}}{a_{*}^{2}}\right)>0 .
$$

(iii) For $q \in\left[0, a_{*}\right), \rho\left(q^{2}\right) q$ is a Lipschitz function of $q$ with Lipschitz constant $\rho_{0}$, as one may easily derive from (ii). Now, suppose $y, z \in \mathbb{R}^{n}, n \geqslant 2$, and $|y|,|z|<a_{*}$; then

$$
\begin{aligned}
\left|\rho\left(|y|^{2}\right) y-\rho\left(|z|^{2}\right) z\right|^{2}= & \left(\rho\left(|y|^{2}\right)|y|-\rho\left(|z|^{2}\right)|z|\right)^{2} \\
& +2 \rho\left(|z|^{2}\right) \rho\left(|y|^{2}\right)(|z||y|-y \cdot z) \\
\leqslant & \left.\rho_{0}^{2}|| y|-| z\right|^{2}+2 \rho_{0}^{2}(|z||y|-y \cdot z) \\
= & \rho_{0}^{2}|y-z|^{2} .
\end{aligned}
$$

Thus, $\rho\left(|y|^{2}\right) y$ is a Lipschitz function of $y$ in $\mathbb{R}^{n}$ provided that $|y| \leqslant a_{*}$. 
We now show that $J^{\prime}$ is Lipschitz continuous and monotone in $K_{\delta}$. For any $u$, $v$, in $K_{\delta}$ and $w$ in $H_{\Gamma_{0}}$,

$$
\begin{aligned}
\left\langle J^{\prime} u-J^{\prime} v, w\right\rangle & =\int_{\Omega}\left[\rho\left(|\nabla u|^{2}\right) \nabla u-\rho\left(|\nabla v|^{2}\right) \nabla v\right] \cdot \nabla w d x \\
& \leqslant \int_{\Omega} \rho_{0}|\nabla u-\nabla v||\nabla w| d x \\
& \leqslant \rho_{0}\|u-v\|\|w\|
\end{aligned}
$$

Thus

$$
\left\|J^{\prime} u-J^{\prime} v\right\|^{*} \leqslant \rho_{0}\|u-v\|,
$$

where $\|\cdot\|^{*}$ is the norm on $H_{\Gamma_{0}}^{\prime}$. As a consequence, the functional $J$ is continuously differentiable in $K_{\delta}$.

From (ii) and the mean value theorem, we see that for any $u, v \in K_{\delta}, u \neq v$,

$$
\begin{aligned}
\left\langle J^{\prime} u-J^{\prime} v, u-v\right\rangle= & \int_{\Omega}\left(\rho\left(|\nabla u|^{2}\right)|\nabla u|-\rho\left(|\nabla v|^{2}\right)|\nabla v|\right)(|\nabla u|-|\nabla v|) d x \\
& +\int_{\Omega}\left[\rho\left(|\nabla u|^{2}\right)+\rho\left(|\nabla v|^{2}\right)\right](|\nabla u||\nabla v|-\nabla u \cdot \nabla v) d x \\
= & \left.\int_{\Omega} \int_{0}^{1}(\rho(t) t)^{\prime}\right|_{t=s|\nabla u|+(1-s)|\nabla v|} d s(|\nabla u|-|\nabla v|)^{2} d x \\
& +2 \rho_{\min } \int_{\Omega}(|\nabla u||\nabla v|-\nabla u \cdot \nabla v) d x
\end{aligned}
$$

and hence

$$
\left\langle J^{\prime} u-J^{\prime} v, u-v\right\rangle>0 .
$$

Thus, $J^{\prime}$ is strictly monotone on $K_{\delta}$ and consequently $J$ is strictly convex. As $K_{\delta}$ is bounded, the existence and uniqueness of solutions of (7) and, equivalently, of (9) may be established by appealing to standard theory. Furthermore, we may deduce from (i) and (ii) that

$$
\left\langle J^{\prime} w-J^{\prime} v, u-v\right\rangle \geqslant C(\delta)\|u-v\|^{2}
$$

for all $u, v$ in $K_{\delta}$.

\section{Estimates}

\subsection{Standard Finite Element Method}

Let $T_{h}$ be a triangulation of $\Omega$ and $P_{k}(\Omega)$ be the space of piecewise polynomials of degree $\leqslant k$ in $\Omega$. Let $V_{h} \subset H^{1}(\Omega)$ be the space of linear finite elements with respect to $T_{h}$. Let $u$ be the weak solution of the variational inequality 
problem (9), and let $u_{h}$ be the corresponding finite element solution, that is,

$$
\left\langle J^{\prime} u_{h}, v_{h}-u_{h}\right\rangle \geqslant 0 \text { for all } v_{h} \in K_{\delta}^{h},
$$

where $K_{\delta}^{h}=V_{h} \cap K_{\delta}$ is a finite element subset of $K_{\delta}$. Setting $v=u_{h}$ in (9) and adding it to (13), we get

$$
\begin{aligned}
0 & \leqslant\left\langle J^{\prime} u_{h}, v_{h}-u_{h}\right\rangle+\left\langle J^{\prime} u, u_{h}-u\right\rangle \\
& =\left\langle J^{\prime} u_{h}-J^{\prime} u, v_{h}-u_{h}\right\rangle+\left\langle J^{\prime} u, v_{h}-u\right\rangle .
\end{aligned}
$$

Thus

$$
\left\langle J^{\prime} u-J^{\prime} u_{h}, v_{h}-u_{h}\right\rangle \leqslant\left\langle J^{\prime} u, v_{h}-u\right\rangle .
$$

Now, by the monotonicity of $J^{\prime}$ in $K_{\delta}$ given in (12), we have

$$
\begin{aligned}
C(\delta)\left\|u-u_{h}\right\|^{2} & \leqslant\left\langle J^{\prime} u-J^{\prime} u_{h}, u-u_{h}\right\rangle \\
& =\left\langle J^{\prime} u-J^{\prime} u_{h}, u-v_{h}\right\rangle+\left\langle J^{\prime} u-J^{\prime} u_{h}, v_{h}-u_{h}\right\rangle
\end{aligned}
$$

for all $v_{h}$ in $K_{\delta}^{h}$. Thus, using (11) and (14),

$$
C(\delta)\left\|u-u_{h}\right\|^{2} \leqslant \rho_{0}\left\|u-u_{h}\right\|\left\|u-v_{h}\right\|+\left\langle J^{\prime} u, v_{h}-u\right\rangle .
$$

Integrating the term $\left\langle J^{\prime} u, v_{h}-u\right\rangle$ in (15) by parts and applying the Hölder inequality, we obtain

$$
\begin{aligned}
C(\delta)\left\|u-u_{h}\right\|^{2} \leqslant & \rho_{0}\left\|u-u_{h}\right\|\left\|u-v_{h}\right\| \\
& \left.+\| \nabla \cdot\left(\rho|\nabla u|^{2}\right) \nabla u\right)\left\|_{L^{p^{\prime}(\Omega)}}\right\| u-v_{h} \|_{L^{p}(\Omega)}
\end{aligned}
$$

where $p \geqslant 2$ and $1 / p+1 / p^{\prime}=1$. To see that the term $\|\nabla \cdot(\rho \nabla u)\|_{L^{p^{\prime}}}$ is bounded when $u \in W^{2, p}(\Omega)$, first note that

$$
|\nabla \cdot(\rho \nabla u)| \leqslant\left|\rho \nabla^{2} u\right|+\left.2\left|\rho^{\prime}\right| \nabla u\right|^{2}|||\nabla u|^{2}|| \sum_{i, j=1}^{N} \frac{\partial^{2} u}{\partial x_{t} \partial x_{j}} \mid .
$$

Recalling that $|\nabla u|^{2} \leqslant \delta$ a.e. in $\Omega$, we have from (i) and (ii)

$$
\begin{aligned}
\|\nabla \cdot(\rho \nabla u)\|_{L^{p^{\prime}}} & \leqslant C\left(\rho_{0}\|u\|_{2, p^{\prime}}+\frac{\rho_{0}}{a_{0}^{2}} \delta^{2}\|u\|_{2, p^{\prime}}\right) \\
& \leqslant C\|u\|_{2, p^{\prime}} \leqslant C\|u\|_{2, p},
\end{aligned}
$$

since $p \geqslant 2$. Here $C$ denotes a generic constant. Now from (16)

$$
\left\|u-u_{h}\right\|^{2} \leqslant C^{-1}(\delta)\left[\rho_{0}\left\|u-u_{h}\right\|\left\|u-v_{h}\right\|+\|\nabla \cdot(\rho \nabla u)\|_{L^{p^{\prime}}}\left\|u-v_{h}\right\|_{L^{p}}\right],
$$

so

$$
\begin{aligned}
\left\|u-u_{h}\right\|^{2} \leqslant & \frac{1}{2}\left\|u-u_{h}\right\|^{2}+\frac{1}{2} C^{-2}(\delta) \rho_{0}^{2}\left\|u-v_{h}\right\|^{2} \\
& +C^{-1}(\delta)\left\|u-v_{h}\right\|_{L^{p}} C\|u\|_{2, p},
\end{aligned}
$$


whence

$$
\left\|u-u_{h}\right\|^{2} \leqslant C^{-2}(\delta) \rho_{0}^{2}\left\|u-v_{h}\right\|^{2}+C^{-1}(\delta) C\|u\|_{2, p}\left\|u-v_{h}\right\|_{L^{p}} .
$$

Note that as $\delta \rightarrow a_{*}, C^{-1}(\delta) \rightarrow \infty$ and thus the error estimate becomes invalid when the flow is no longer subsonic.

Let $\Pi_{h}$ denote the linear interpolation operator on $C^{0}(\bar{\Omega})$, with respect to the triangulation $T_{h}$, i.e., for $v \in C^{0}(\bar{\Omega})$,

$$
\left.\Pi_{h} v\right|_{T} \in P_{1}(T) \text { for all } T \text { in } T_{h}
$$

and $\Pi_{h} v(P)=v(P)$ at all nodal points $P$ in $T_{h}$.

As $u \in K_{\delta}$ and $W^{1, \infty}(\Omega) \hookrightarrow C^{0}(\bar{\Omega}), \Pi_{h} u$ is well defined. However, as $\Pi_{h} u \notin$ $K_{\delta}^{h}$ in general, we cannot set $v_{h}$ in (18) as $\Pi_{h} u$ to obtain an optimal estimate. Instead, we shall construct an element in $K_{\delta}^{h}$ with which approximation results may be obtained easily.

From standard approximation theory, we have, for $v$ in $W^{2, p}(\Omega), p>2$,

$$
\left|\nabla\left(\Pi_{h} v-v\right)(x)\right| \leqslant C h^{1-2 / p}\|v\|_{2, p}
$$

for a.e. $x$ in $\Omega$, where $C$ is a constant independent of $v$ and $h$. If we restrict $v$ to $K_{\delta}$, then

$$
\left|\nabla\left(\Pi_{h} v\right)(x)\right| \leqslant \delta+C h^{1-2 / p}\|v\|_{2, p}
$$

for a.e. $x$ in $\Omega$. Based on this inequality, and motivated by the analysis of $[8,12]$, we introduce the element

$$
r_{h} u=\frac{\delta \Pi_{h} u}{\delta+C h^{1-2 / p}\|u\|_{2, p}},
$$

where $p>2$. Note that as $h \rightarrow 0, r_{h} u \rightarrow \Pi_{h} u$. Also,

$$
\left|r_{h} u\right| \leqslant \delta \quad \text { a.e. in } \Omega \text {. }
$$

Thus, $r_{h} u \in K_{\delta}^{h}$. Moreover,

$$
\left\|u-r_{h} u\right\|=O\left(h+h^{1-2 / p}\right)
$$

and

$$
\left\|u-r_{h} u\right\|_{L^{p}}=O\left(h^{2}+h^{1-2 / p}\right) .
$$

Thus, using (20) and (21) in (18), we have the estimate

$$
\left\|u-u_{h}\right\|=O\left(h^{1 / 2-1 / p}\right) \text { for } u \in W^{2, p}(\Omega), p>2 .
$$

REMARKs. 1. The suboptimality of this result relative to the $O(h)$ result for linear thoery originates from the "incomplete" approximation theory for the subset $K_{\delta}$ rather than the nonoptimality of the abstract error estimate.

2. One can obtain an estimate without making the above regularity assumption on $u$ as follows: The second term on the right-hand side of (15) is bounded by $C\left\|J^{\prime} u\right\|^{*}\left\|v_{h}-u\right\|$ and the first term on the right-hand side is, as before, bounded 
by

Thus,

$$
\frac{1}{2} C(\delta)\left\|u-u_{h}\right\|^{2}+\frac{1}{2} \rho_{0}^{2} C^{-1}(\delta)\left\|u-v_{h}\right\|^{2}
$$

$$
\left\|u-u_{h}\right\|^{2} \leqslant \rho_{0}^{2} C^{-2}(\delta)\left\|u-v_{h}\right\|^{2}+C C^{-1}(\delta)\left\|v_{h}-u\right\| .
$$

With this approach, we do not need to impose the regularity condition $u \in$ $W^{2, p}(\Omega)$ for $p \geqslant 2$. However, the error estimate is necessarily suboptimal because of the presence of the second term on the right-hand side of (23).

3. With the establishment of the error estimates, it is easy to prove the convergence of the finite element approximation $u_{h}$ to the solution $u$.

\subsection{Dual Finite Element Method}

The error estimate (22) derived in the previous section is useful in proving the convergence of the finite element approximation. However, even if we assume $u$ to be in $W^{2, \infty}(\Omega)$, this error estimate would still be suboptimal due to the inadequacy of $K_{\delta}^{h}$. More specifically, with this strong smoothness assumption, the bound on $\left\|u-u_{h}\right\|$ would be of $O\left(h^{1 / 2}\right)$, which is inferior to the standard $O(h)$ estimates obtained for many other problems. The presence of the nonlinearity and critical flow degeneracy at $q=a^{*}$ may lead one to admit the possible suboptimality, but numerical evidence suggests that the energy error for $q<a^{*}$ is actually $O(h)$ [4]. In this section, we follow a successful approach used for elastoplastic torsion problems $[8,9,12]$ to derive an optimal estimate for the dual finite element approximation for subsonic flow. (By working with the velocity directly rather than the potential, the previous difficulty-Remark 1, Section 2.1 - can be circumvented.)

Let $u \in K_{\delta}$ be the unique solution of the variational inequality problem (9) and let $v$ be an element of $K_{\delta}$. We introduce as new variables for a dual formulation

$$
\boldsymbol{\xi}=\left(\frac{\partial u}{\partial y},-\frac{\partial u}{\partial x}\right) \text { and } \boldsymbol{\eta}=\left(\frac{\partial v}{\partial y},-\frac{\partial v}{\partial x}\right) .
$$

Clearly, the subsonic constraint becomes

$$
\begin{gathered}
\eta \equiv|\eta|=|\nabla v| \leqslant \delta, \text { so }|\eta| \in L^{\infty}(\Omega), \\
\eta \in L_{\delta} \equiv\left\{\zeta \in L^{2}(\Omega) \times L^{2}(\Omega) ; \zeta \leqslant \delta\right\} .
\end{gathered}
$$

The subsonic constraint in this formulation appears as a constraint on the magnitude of the elements in $L_{\delta}$. This seemingly trivial change is actually an important step in establishing the optimal error estimate. While we cannot guarantee that the linear interpolant of elements of $K_{\delta}$ be in $K_{\delta}^{h}$, a fact leading us to a suboptimal estimate, the constraint in $L_{\delta}$ involves no derivative and the linear interpolant of any element in $L_{\delta}$ will belong to the approximating space $L_{h}$ 
of $L_{\delta}$ defined in the next section. This then allows us to utilize the optimal approximation result in our error analysis. The far-field boundary condition $\phi=g_{0}$ on $\Gamma_{0}$ may be restated in terms of $\partial g_{0} / \partial \tau$ where $\tau=\left(n_{2},-n_{1}\right)$ is the tangent vector. We first embed this condition in the space for the dual problem and then subsequently consider using a Lagrange multiplier for the boundary constraint. An error estimate is finally derived for the finite element approximation of the dual formulation with multiplier treatment of the boundary condition.

We first show that the vector $\xi$ defined above is a solution of the following dual variational inequality problem. Find $\xi \in L_{\delta} \cap H$ such that

$$
\int_{\Omega} \rho\left(\xi^{2}\right) \xi \cdot(\eta-\xi) d x \geqslant 0 \text { for all } \eta \text { in } L_{\delta} \cap H,
$$

where

$$
H=\left\{\eta \in L^{2}(\Omega) \times L^{2}(\Omega) ; \int_{\Omega} \eta \cdot \nabla w d x=\int_{\Gamma_{0}}-\frac{\partial g_{0}}{\partial \tau} w d s \text { for all } w \in H_{\Gamma_{1}}\right\} .
$$

For notational convenience, henceforth we shall write $H_{\Gamma}$ for $H_{\Gamma_{1}}$. Now

$$
\int_{\Omega} \rho\left(|\nabla u|^{2}\right) \nabla u \cdot(\nabla v-\nabla u) d x=\int_{\Omega} \rho\left(\xi^{2}\right) \xi \cdot(\eta-\xi) d x
$$

follows directly from the definition of $\xi$ and $\eta$ and the property

$$
\boldsymbol{\xi} \cdot \boldsymbol{\eta}=\frac{\partial u}{\partial y} \frac{\partial v}{\partial y}+\left(-\frac{\partial u}{\partial x}\right)\left(-\frac{\partial v}{\partial x}\right)=\nabla u \cdot \nabla v .
$$

Furthermore, since $\eta=(\partial v / \partial y,-\partial v / \partial x)$ and $v=g_{0}$ on $\Gamma_{0}\left(v \in K_{\delta}\right)$, then for any $w$ in $H_{\Gamma}$,

$$
\begin{aligned}
\int_{\Omega} \eta \cdot \nabla w d x & =\int_{\Gamma_{0}} w \eta \cdot \mathbf{n} d s-\int_{\Omega}(\nabla \cdot \eta) w d x \\
& =\int_{\Gamma_{0}}-w \frac{\partial g_{0}}{\partial \tau} d s .
\end{aligned}
$$

Thus, $\eta \in H$ and so $\xi$ is a solution of the dual variational inequality (24).

In fact, the converse is also true, i.e., if $\xi$ is a solution of (24), then one may find function $u$ satisfying (9) and such that curl $u=\xi$ (see (27) below). To show this, we take $\eta \in L_{\delta} \cap H$. Then, for all $w \in H_{\Gamma}$, we again have (26). Now, for $w$ in $H_{0}^{1}(\Omega) \subset H_{\Gamma},(26)$ is reduced to $\int_{\Omega}(\nabla \cdot \eta) w d x=0$ which implies $\nabla \cdot \eta=0$ a.e. in $\Omega$. Consequently, we have $\int_{\Gamma_{0}} w \eta \cdot \mathbf{n} d s=\int_{\Gamma_{0}}-w \partial g_{0} / \partial \tau d s$ for all $w \in H_{\Gamma}$; therefore,

$$
\boldsymbol{\eta} \cdot \mathbf{n}=-\partial g_{0} / \partial \tau \text { a.e. on } \Gamma_{0} .
$$

Next, observe that as $\Omega$ is simply connected, there exists a function $v$ in $H^{1}(\Omega)$, unique up to an additive constant, such that

$$
\eta=\operatorname{curl} v \equiv\left(\frac{\partial v}{\partial y},-\frac{\partial v}{\partial x}\right)
$$


(so that $\nabla \cdot \eta=0$ is satisfied automatically) and that by (27)

$$
-\frac{\partial g_{0}}{\partial \tau}=\eta \cdot \mathbf{n}=\frac{\partial v}{\partial y} n_{1}-\frac{\partial v}{\partial x} n_{2}=-\nabla v \cdot \tau=-\frac{\partial v}{\partial \tau} .
$$

To fix $v$, we may take $v=g_{0}$ on $\Gamma_{0}$ to obtain $v \in K_{\delta}$. In particular, we may set $\eta$ to $\xi$, and the corresponding $v$ to $u$. Thus, in view of equation (25), we have shown the converse.

Formulation (24) is, in some sense, an unconventional mixed problem, as most mixed problems involve a Lagrange multiplier and may be related to a saddle-point problem. This situation is clarified by noting that the space $H$ involves a constraint arising from the far-field boundary condition and may be relaxed with the aid of a Lagrange multiplier.

In order to relax the constraint $\int_{\Omega} \eta \cdot \nabla w d x=\int_{\Gamma_{0}}-\left(\partial g_{0} / \partial \tau\right) w d s$ for $w$ in $H_{\Gamma}$, we introduce the multiplier $\chi \in H_{\Gamma}$ and consider the variational problem using Lagrange multipliers: find the pair $\langle\xi, \chi\rangle$ in $\left(L_{\delta} \cap H\right) \times H_{\Gamma}$ such that

$$
\int_{\Omega} \rho\left(\xi^{2}\right) \xi \cdot(\eta-\xi) d x \geqslant \int_{\Omega} \nabla \chi \cdot(\eta-\xi) d x \quad \text { for all } \eta \text { in } L_{\delta} .
$$

This formulation (29) will be useful later when we estimate the error of the finite element approximation.

We have the following theorem:

THEOREM. The mixed variational inequality problem (24) is equivalent to the variational problem (29).

Proof. Let $(\xi, \chi)$ be a solution of (29). By restricting $\eta$ in (29) to the set $L_{\delta} \cap H$, we have

$$
\int_{\Omega} \nabla x \cdot(\eta-\xi) d x=0
$$

since $\chi \in H_{\Gamma}$. Thus, $\xi$ is a solution of (24). Next, let $\xi$ be a solution of the mixed problem (24). To prove the existence of $\chi$ in $H_{\Gamma}$ such that (29) holds for all $\eta$ in $L_{\delta}$, first observe that $\xi$ is the solution of the minimization problem: find $\xi \in L_{\delta} \cap H$ such that

$$
J_{1}(\xi)=\min J_{1}(\eta), \quad \eta \in L_{\delta} \cap H,
$$

where

$$
J_{1}(\eta)=\int_{\Omega} \int_{0}^{\eta^{2}} \frac{1}{2} \rho(t) d t d x
$$

We shall prove the desired inequality by contradiction. Suppose that for all $\chi$ in $H_{\Gamma}$, there exists $\eta$ in $L_{\delta}$ such that

$$
J_{1}(\eta)-\int_{\Omega} \nabla \chi \cdot \eta d x<J_{1}(\xi) .
$$


Clearly, $\eta \neq \xi$, otherwise we have an immediate contradiction. This and the uniqueness of $\xi$ imply that $J_{1}(\xi)<J_{1}(\eta)$ for all $\chi$. Now, let $\left\{\chi_{k}\right\}$ be a sequence of functions in $H_{\Gamma}$ converging to 0 , and let $\left\{\eta_{k}\right\}$ be the corresponding sequence in $L_{\delta}$. As $L_{\delta}$ is a compact set in $L^{2}(\Omega) \times L^{2}(\Omega)$, there is a convergent subsequence $\left\{\boldsymbol{\eta}_{k}\right\}$ converging to some $\boldsymbol{\eta}$ in $L_{\delta}$. From (31), we have

$$
J_{1}\left(\eta_{k}\right)-\|\nabla \chi\| \delta<J_{1}(\xi) .
$$

Taking the limit $k \rightarrow \infty$, we have $J_{1}(\eta)<J_{1}(\xi)$ which contradicts the fact that $\xi$ is a minimum point of (30). Thus, we have shown the existence of $\chi$ in $H_{\Gamma}$ such that (29) holds for all $\eta$ in $L_{\delta}$, since $\int_{\Omega} \xi \cdot \nabla \chi d x=0$.

REMARK. The variational problem (29) may be shown to be equivalent to the saddle point problem: find $\langle\xi, \chi\rangle \in L_{\delta} \times H_{\Gamma}$ such that

$$
L(\xi, w) \leqslant L(\xi, \chi) \leqslant L(\eta, \chi)
$$

for all $(\eta, w)$ in $L_{\delta} \times H_{\Gamma}$, where

$$
L(\eta, w)=\int_{\Omega} \int_{0}^{\eta^{2}} \frac{1}{2} \rho(t) d t d x-\int_{\Omega} \eta \cdot \nabla w d x+\int_{\Gamma_{0}} \frac{\partial g_{0}}{\partial \tau} w d s .
$$

\subsection{Error Estimate for Dual Method}

Let us now consider the approximate dual method. Given a triangulation $T_{h}$ of finite elements of $\Omega$, we define the following approximation spaces:

$$
\begin{aligned}
& \Lambda_{h}=\left\{\zeta_{h} \in L^{2}(\Omega) \times L^{2}(\Omega) ;\left.\zeta_{h}\right|_{T} \in P_{0}(T) \text { for } T \text { in } T_{h}\right\} \\
& L_{h}=\left\{\zeta_{h} \in \Lambda_{h} ;\left|\zeta_{h}\right| \leqslant \delta \text { a.e. in } \Omega\right\} ; \\
& V_{h}=\left\{W_{h} \in C^{0}(\bar{\Omega}) ;\left.w_{h}\right|_{T} \in P_{1}(T) \text { for } T \text { in } T_{h}, w_{h}=0 \text { on } \Gamma\right\}
\end{aligned}
$$

and

$$
H_{h}=\left\{\zeta_{h} \in \Lambda_{h} ; \int_{\Omega} \zeta_{h} \cdot \nabla w_{h} d x=0 \text { for all } w_{h} \text { in } V_{h}\right\}
$$

The approximation to the dual problem (24) takes the form: find $\xi_{h} \in L_{h} \cap H_{h}$ such that

$$
\int_{\Omega} \rho\left(\xi_{h}^{2}\right) \xi_{h} \cdot\left(\eta_{h}-\xi_{h}\right) d x \geqslant 0
$$

for all $\eta_{h}$ in $L_{h} \cap H_{h}$. If $(\xi, \chi)$ is a solution of the variational problem (29), then setting $\eta=\xi_{h}\left(\in L_{h} \cap H_{h} \cap L_{\delta}\right)$ in (29), we have

$$
\int_{\Omega} \rho\left(\xi^{2}\right) \xi \cdot\left(\xi_{h}-\xi\right) d x \geqslant \int_{\Omega} \nabla \chi \cdot\left(\xi_{h}-\xi\right) d x .
$$


Adding the inequalities (33) and (34), we have

$$
\begin{aligned}
& \int_{\Omega} \rho\left(\xi_{h}^{2}\right) \xi_{h} \cdot\left(\eta_{h}-\xi_{h}\right) d x+\int_{\Omega} \rho\left(\xi^{2}\right) \xi \cdot\left(\xi_{h}-\xi\right) d x \\
& \quad \geqslant \int \nabla \chi \cdot\left(\xi_{h}-\xi\right) d x
\end{aligned}
$$

Using the monotonicity pioperty (iii) of the function $\rho\left(t^{2}\right) t$ and (35), we have

$$
\begin{aligned}
& C(\delta)\left\|\xi_{h}-\xi\right\|_{L^{2} \times L^{2}}^{2} \leqslant \int_{\Omega}\left(\rho\left(\xi_{h}^{2}\right) \xi_{h}-\rho\left(\xi^{2}\right) \xi\right) \cdot\left(\xi_{h}-\xi\right) d x \\
& \leqslant \int_{\Omega} \rho\left(\xi_{h}^{2}\right) \xi_{h} \cdot\left(\xi_{h}-\xi\right) d x+\int_{\Omega} \rho\left(\xi_{h}^{2}\right) \xi_{h} \cdot\left(\eta_{h}-\xi_{h}\right) d x \\
&-\int_{\Omega} \nabla x \cdot\left(\xi_{h}-\xi\right) d x \\
& \leqslant \int_{\Omega} \rho\left(\xi_{h}^{2}\right) \xi_{h} \cdot\left(\eta_{h}-\xi_{h}\right) d x-\int_{\Omega} \nabla\left(x-w_{h}+w_{h}\right) \cdot\left(\xi_{h}-\xi\right) d x \\
& \leqslant \int_{\Omega} \rho\left(\xi_{h}^{2}\right) \xi_{h} \cdot\left(\eta_{h}-\xi_{h}\right) d x-\int_{\Omega} \nabla w_{h} \cdot\left(\xi_{h}-\xi\right) d x \\
&+\frac{1}{2} C(\delta)\left\|\xi_{h}-\xi\right\|_{L^{2} \times L^{2}}^{2}+\frac{1}{2} C^{-1}(\delta) \int_{\Omega}\left|\nabla\left(x-w_{h}\right)\right|^{2} d x
\end{aligned}
$$

where $w_{h}$ is an arbitrary element in $V_{h}$.

Now, $\xi_{h} \in H_{h}$ and $\xi \in H$, so $\int_{\Omega} \nabla w_{h} \cdot\left(\xi_{h}-\xi\right) d x=0$. Moreover, as $\left.\xi_{h}\right|_{T} \in$ $P_{0}(T) \times P_{0}(T)$, so

$$
\left.\rho\left(\xi_{h}^{2}\right)\right|_{T} \in P_{0}(T)
$$

Thus

$$
\left.\rho\left(\xi_{h}^{2}\right) \xi_{h}\right|_{T} \in P_{0}(T) \times P_{0}(T) \text { for all } T \text { in } T_{h}
$$

and hence $\rho\left(\xi_{h}^{2}\right) \xi_{h} \in \Lambda_{h}$. Now, $L_{h}$ is a closed, convex subset of $L^{2}(\Omega) \times L^{2}(\Omega)$. Thus one may define an orthogonal projection $R_{h}$ from $L^{2}(\Omega) \times L^{2}(\Omega)$ onto $L_{h}$. Setting $\eta_{h}$ in (36) as $R_{h} \xi$, we clearly have

$$
\int \rho\left(\xi_{h}^{2}\right) \xi_{h} \cdot\left(R_{h} \xi-\xi\right) d x=0 .
$$

Finally, let $\Pi_{h}: C^{0}(\bar{\Omega}) \rightarrow V_{h}$ be the $V_{h}$-interpolation operator on $T_{h}$. The regularity assumption $\chi \in H^{2}(\Omega) \rightarrow C^{0}(\bar{\Omega})$ allows us to conclude $\Pi_{h} \chi \in V_{h}$ and

$$
\left\|\Pi_{h} \chi-\chi\right\| \leqslant C h\|\chi\|_{2,2} \text {. }
$$

Setting $w_{h}=\Pi_{h} \chi$ in (36), we then have

$$
\left\|\xi_{h}-\xi\right\|_{L^{2} \times L^{2}}^{2} \leqslant C C^{-2}(\delta) h^{2}\|\chi\|_{2,2}^{2}
$$


or

$$
\left\|\xi_{h}-\xi\right\|_{L^{2} \times L^{2}}=O(h / C(\delta)) .
$$

We summarize this result in the following theorem:

THEOREM. Let $\xi$ be the solution of the mixed variational inequality problem (24) and $\xi_{h}$ be the finite element solution of the approximation problem (33). Let $\chi$ be the Lagrange multiplier in (29) corresponding to (24). Under the regularity assumption $\chi \in H^{2}(\Omega)$, we have $\left\|\xi-\xi_{h}\right\|_{L^{2} \times L^{2}}=O(h / C(\delta))$.

REMARKS. 1. In the proof above, the multiplier formulation rather than the original dual formulation is used because $\xi_{h}$ is not necessarily in $H$. The multiplier relaxes the restriction for $\xi_{h}$.

2. Notice that, in the above proof, the regularity of $\xi$ is not needed. Instead, the regularity of the multiplier $\chi$ is required.

3. In practice, it is easier to implement and numerically solve the saddle point problem (32) than the variational problem (29). Since these problems are equivalent, the error estimate established is also applicable to the numerical solution of (32).

\section{Acknowledgements}

This research has been supported in part by the Department of Energy and the Office of Naval Research.

\section{References}

[1] L. Bers, Mathematical aspects of subsonic and transonic gas dynamics (Interscience, New York, 1958).

[2] H. Brezis and G. Stampacchia, "The hodograph method in fluid mechanics in the light of variational inequalities", Arch. Rational Mech. Anal. 61, 1 (1976), 1-18.

[3] M. Bristeau, R. Glowinski, J. Periaux, P. Perrier, O. Pirfonneau, and G. Poirier, "Transonic flow simulation by finite element and least square methods", in Finite elements in fluids Vol. 4, R. H. Gallagher et al (eds.) (Wiley, New York, 1982).

[4] G. F. Carey and T. T. Pan, "Computation of subcritical compressible flows", Computers and Fluids 9 (1981), 33-41.

[5] G. F. Carey and T. T. Pan, "Shock free redesign using finite elements", Comm. Appl. Num. Meth. (in press) (1985).

[6] H. Dinh and G. F. Carey, "Approximate analysis of regularized compressible flow", J. Nonlin. Anal. (in review) (1984). 
[7] H. Dinh and G. F. Carey, "Some results concerning approximation of regularized compressible flow", Internat. J. Numer. Methods Fluids 5 (1985), 299-302.

[8] R. S. Falk, "Approximate solutions of some variational inequalities with order of convergence estimates", Ph.D. Thesis, Cornell University, Ithaca, New York, 1971.

[9] R. S. Falk and B. Mercier, "Error estimates for elasto-plastic problems", RAIRO Anal. Numer. 11, 2 (1977), 135-144.

[10] R. Finn and D. Gilbarg, "Asymptotic behavior and uniqueness of plane subsonic flows", Comm. Pure Appl. Math. 10 (1957), 23-63.

iiij $\bar{P}$. Germain, "Ecoulements transsoniques homogènes", 5 Prog. in aeronautical sci. (1964), 143-273.

[12] R. Glowinski, J. L. Lions and R. Tremoliers, "Numerical analysis of variational inequalities", (North Holland, Amsterdam, 1982).

[13] K. G. Guderley, The theory of transonic flow (Addison-Wesley, 1962) (English Translation).

[14] M. Hafez, C. Wellford, E. Murman, "Mixed finite element methods and dual iterative methods for transonic flow", in Finite elements and fluids, Vol. 3, R. H. Gallagher et al (eds.) (Wiley, New York, 1978).

[15] K.-T. Li and A. X. Huang, "Solvability of the partial differential equation satisfied by the stream function in compressible flow and the error bound in the finite element solution", in Finite element flow analysis, Tadahiko Kawai (ed.) (North-Holland, Amsterdam, 1982) 387-394.

[16] C. S. Morawitz, "On the non-existence of continuous transonic flows past profiles," Parts I, II Comm. Pure Appl. Math. 10 1957, 45-68.

[17] A. H. Shapiro, The dynamics and thermodynamics of compressible fluid flow, Vols. 1, 2. (Ronald Press, New York, 1953).

[18] S. F. Shen, "Transonic aerodynamics", in Finite elements and flutds, Vol. 3, R. H. Gallagher et al (eds.) (Wiley, New York, 1978).

[19] R. von Mises, Mathematical theory of compressible fluid flow (Academic Press, New York, 1958). 